\title{
Femoral quadriceps neuromuscular electrical stimulation after total knee arthroplasty: a systematic review
}

\author{
Estimulação elétrica neuromuscular do quadríceps após artroplastia total de joelho: \\ uma revisão sistemática
}

\author{
Helena Bruna Bettoni Volpato ${ }^{1}$, Paulo Szego ${ }^{1}$, Mario Lenza ${ }^{1}$, Silvia Lefone Milan ${ }^{1}$, Claudia Talerman ${ }^{1}$, Mario Ferretti ${ }^{1}$
}

\begin{abstract}
The purpose of this study was to evaluate the effects of neuromuscular electrical stimulation in patients submitted to total knee arthroplasty. This was a systematic review with no language or publication status restriction. Our search was made in Cochrane Library, MEDLINE, Embase and LILACS. Randomized or quasi-randomized clinical trials evaluating neuromuscular electrical stimulation after total knee arthroplasty were included. Four studies with moderate risk of bias and low statistical power were included, totalizing 376 participants. There was no statistically significant difference in knee function, pain and range of motion during 12 month follow-up. This review concluded that neuromuscular electrical stimulation was less effective than traditional rehabilitation in function, muscular strength and range of motion. However, this technique was useful for quadriceps activation during the first days after surgery.
\end{abstract}

Keywords: Arthroplasty, replacement, knee; Knee prosthesis; Knee joint; Osteoarthritis; Physical therapy modalities; Electric stimulation; Quadriceps muscle

\section{RESUMO}

0 objetivo deste estudo foi avaliar os efeitos da estimulação elétrica neuromuscular em pacientes submetidos à artroplastia total de joelho. Esta foi uma revisão sistemática sem restrição de idioma ou status de publicação. Nossa pesquisa foi realizada na biblioteca Cochrane, MEDLINE, Embase e LILACS. Foram incluídos ensaios clínicos randomizados ou quase-randomizados, avaliando a estimulação elétrica neuromuscular após a artroplastia total de joelho. Quatro estudos com risco de viés moderado e de baixo poder estatístico foram incluídos, totalizando 376 participantes. Não houve diferença estatisticamente significativa na função do joelho, dor e amplitude de movimento durante 12 meses de seguimento. Esta avaliação concluiu que a estimulação elétrica neuromuscular foi menos eficaz do que a reabilitação tradicional na função, força muscular e amplitude de movimento. No entanto, essa técnica é útil para a ativação do quadríceps durante os primeiros dias após a cirurgia.

Descritores: Artroplastia de joelho; Prótese do joelho; Articulação do joelho; Osteoartrite; Modalidades de fisioterapia; Estimulação elétrica; Músculo quadríceps

\section{INTRODUCTION}

Osteoarthritis is a degenerative joint disease characterized by reduction of articular cartilage in some areas, and can be related to bone hypertrophy (osteophytes and subchondral bone sclerosis) resulting from biochemical alterations and biomechanical stresses. It is estimated that $75 \%$ of the population aged over 65 years is affected by this disease, with a higher prevalence in women, mostly at the knee joint. ${ }^{(1,2)}$

All the above-mentioned alterations result in quality of life reduction due to functional limitation. Knee arthroplasty is a common and effective intervention for knee osteoarthritis treatment when the clinical therapy is unsuccessful. Its applicability grew $73 \%$ at the last 10 years and it is expected that its indication rises more than $600 \%$ (3.48 million procedures) until $2030 .^{(3)}$

Such percent growth in total knee arthroplasty (TKA), first introduced in the 1960s, is due to pain relief and range of motion (ROM) gain. TKA main goal is to reestablish the patient's joint compromised function. This is why TKA is considered as one of the most

\footnotetext{
${ }^{1}$ Hospital Israelita Albert Einstein, São Paulo, SP, Brazil.

Corresponding author: Mario Ferretti - Avenida Albert Einstein 627/701, 3rd floor, building A1 - Morumbi - Zip code: 052652-900 - São Paulo, SP, Brazil - Phone: (55 11) 3078-7945 E-mail: mario.ferretti@einstein.br
}

Received on: Apr 18, 2014 - Accepted on: Feb 8, 2015

DOI: 10.1590/S1679-45082015RW3140 
important surgery developments of this century. On the United States alone, currently nearly 140 thousand TKAs are performed each year. However, surgery itself is not capable to restore the patient's complete functionality. Physical therapy is an integral part of treatment, yielding the best results regarding postoperative pain, physical function and quality of life. ${ }^{(4)}$

As such, early rehabilitation start following TKA significantly benefits joint mobility and muscle stretch gain, favoring important quality of life gains and preventing postoperative complications. Apart from the primary benefits, the immediate rehabilitation could reduce hospital stay and improve the joint functional ability in the short and medium-run. ${ }^{(5)}$ Another study showed joint pain relief and gait velocity and cadence improvement. ${ }^{(6)}$

Labraca et al. ${ }^{(2)}$ showed that, despite the few scientific evidences, isometric and isotonic exercises designed for quadriceps strengthening between zero and $30^{\circ}$ of flexion, ROM gain and inferior limb muscle stretching are usually employed with good results.

Other studies showed that postoperative weakness, muscle atrophy and knee function alterations are common during the first 4 weeks after surgery, causing a quadriceps strength déficit compared to the contralateral limb that reaches a $18 \%$ reduction. ${ }^{(7,8)}$

Although the neurophysiologic mechanisms for quadriceps muscle voluntary activation déficits are not fully understood, spinal reflex activity from swelling or pain in the knee joint may change afferent input from the injured joint and result in diminished efferent motor drive to the quadriceps muscle (also referred to as "reflex inhibition") that reduces muscle strength. ${ }^{(9,10)}$

One of the measures to reduce voluntary activation deficits and prevent muscle atrophy after TKA is the neuromuscular electrical stimulation (NMES) as an adjuvant restoring normal knee function. ${ }^{(9)}$

\section{OBJECTIVE}

Based on the previously mentioned literature, the objective of this review was to systematically evaluate the effects (benefits and harms) of neuromuscular electrical stimulation in patients who underwent to total knee arthroplasty.

\section{METHODS}

\section{Data sources and searches}

Institutional review board approval (number 1,593-12) was obtained to perform this systematic review. The study was registered at - International Prospective Register of Systematic Reviews (Prospero), protocol CRD42013005491.

\section{Types of studies}

We included randomized or quasi-randomized (in which participants therapy-allocation was not strictly random, i.e., using hospital register number, alternation, medical file number etc.) clinical trials evaluating physical therapy interventions with NMES after TKA.

\section{Types of participants}

We included studies that evaluated (skeletally mature) adults who underwent rehabilitation with NMES after TKA.

\section{Types of intervention}

All physical therapy interventions used at post-TKA treatment associated with NMES were considered. We compared NMES with physical therapy rehabilitation.

Studies comparing non-physical therapy-related or specific formation rehabilitation techniques (including Mulligan, Maitland, Pilates etc.) were excluded.

\section{Types of outcome measures}

Primary outcomes included function or disability evaluation. The inferior limb functional outcome was evaluated according validated questionnaires, such as the knee dysfunction-specific Lysholm Knee Scoring Scale, ${ }^{(11)}$ including joint symptoms evaluation.

Quality of life (evaluated by 36-Item Short Form Health Survey - SF-36), ${ }^{(12)}$ and treatment failure (prosthesis loosening) were also observed.

Secondary outcomes included pain, with pain reports evaluated according to validated scales, such as Visual Analogue Scale (VAS) or Numerical Rating Scale (NRS). Range of motion was checked by assessing the joint mobility degrees for both knee flexion and extension. Return to previous activities (work, sport, daily life activities etc.), as well as hospitalization time and costs were considered part of secondary outcomes.

Primary outcomes were evaluated at the following periods: short-term (up to 4 months of treatment) and long-term (over 4 months of treatment) follow-up.

\section{Research methods for studies identification}

\section{Electronic searches}

Our search was performed at Cochrane Central Register of Controlled Trials - CENTRAL (The Cochrane Library, August 2013 edition), MEDLINE (from 1966 up to August 2013), Embase (from 1974 up to August 2013) and LILACS (from 1982 up to August 2013). We also searched Current Controlled Trials (at 
http://www.controlled-trials.com) and Clinical Trials (at www.clinicaltrials.gov) for ongoing and recently completed studies. There were no language or publication status-based restrictions.

At MEDLINE (PubMed), a specific filter (sensitivity and maximum precision version) for randomized clinical trials identification was combined to a specific subject strategy. ${ }^{(13)}$ Search strategies were also performed at The Cochrane Library (Wiley InterScience), Embase (Elsevier) and LILACS (Bireme) as described in appendix 1.

\section{Searching other resources}

We checked the reference lists of articles and reviews for possible relevant studies.

\section{Study selection}

Two authors independently selected potential eligible titles and abstracts to be included on this review and extracted data using pre-piloted form. Any disagreements were resolved by discussion and, when necessary, with adjudication by a third author. Authors were not blinded to journal and/or authors.

\section{Data extraction and quality assessment Data extraction and management}

Two authors collected the following data using a prepiloted data extraction form: study methodology characteristics, including study design and duration and the protocol publication prior patient recruitment; financing sources and register details; study participants characteristics, with study site, number of enrolled participants, number of evaluated participants, inclusion criteria, exclusion criteria, participants' age, prosthesis types and surgical techniques; study intervention characteristics, including intervention time, physical therapy intervention and other co-intervention types; study result characteristics, including follow-up time, loss at follow-up and outcome measures; and methodological domains, as described below at the risk of bias evaluation section. Any discrepancies were settled by a third reviewer. Two review authors inputted the data at Review Manager ${ }^{\mathrm{TM}}$.

\section{Assessment of risk of bias in the included studies}

The risk of bias of the included studies was independently evaluated by two authors. As recommended by The Cochrane Collaboration's "Risk of bias" tool, ${ }^{(14)}$ the following domains were assessed: random sequence generation; allocation concealment; blinding of participants and personnel; blinding of outcome assessment; incomplete outcome data; selective reporting; other bias (e.g., great imbalance between participants groups and risk of bias associated with testers and other caretakers' inexperience).

Each individual criterion was deemed as presenting low risk of bias, high risk of bias and uncertain risk of bias (lack of information or uncertainties regarding potential bias). Discrepancies between authors were solved based on a consensus.

\section{Data synthesis and analysis Measures of treatment effect}

The risk ratio with a $95 \%$ confidence interval $(95 \% \mathrm{CI})$ was calculated for dichotomous variables. Continuous variables results were expressed as mean differences (MD) with $95 \%$ CI.

\section{Unit of analysis issues}

At the studies included in this review, randomization was based on individual participants. Exceptionally, as in clinical trials including patients with bilateral knee prostheses, data may have been laterally evaluated rather than by individual patients. During the analysis of questions lacking proper corrections, the presentation of such clinical trials data was considered only when discrepancies between analysis units and randomization were small. After data compilation, a sensitivity analysis was performed to examine the effects of the incorrectly evaluated clinical trials in the studies correctly addressed.

\section{Dealing with missing data}

The data on outcome were extracted for all randomized patients. When required, the primary study authors were contacted to request missing data, with participant number, sampling loss details, uncertainty measures (standard deviation or error) or number of events.

The standard deviation of continuous variables, with no report of such figure, was calculated using $\mathrm{p}$ value and values (95CI\%). ${ }^{(14)}$ The impossibility of sampling loss data obtainment was described at the risk of bias table, including a discussion regarding the potential influence of such data at the results and conclusions of the present review. Sensitivity analysis was applied in order to explore these missing data effects. ${ }^{(15)}$ 


\section{Assessment of heterogeneity}

We assessed the heterogeneity of estimate effects between the included studies by visual inspection of the forest plot and using the $\mathrm{I}^{2}$ statistic.

We quantified the possible magnitude of inconsistency (i.e. heterogeneity) across studies, using the $\mathrm{I}^{2}$ statistic with a rough guide for interpretation as follows: zero to $40 \%$ might not be important; $30 \%$ to $60 \%$ may represent moderate heterogeneity; $50 \%$ to $90 \%$ may represent substantial heterogeneity; and $75 \%$ to $100 \%$ considerable heterogeneity. ${ }^{(14)}$ In cases of considerable heterogeneity (defined as $\mathrm{I}^{2} 75 \%$ ), we explored the data further by comparing the characteristics of individual studies and conducting subgroup analyses.

\section{Assessment of reporting biases}

For meta-analysis with more than ten studies, primary outcomes graphs were draw in order to evaluate the potential publication bias (small studies effects). The presence of bias was also evaluated in small studies, to verify if random intervention events were more beneficial compared to fixed events estimative. ${ }^{(16)}$

\section{Data synthesis}

When appropriate, results of comparable groups of studies were pooled in meta-analysis using the random-effects model as a default. For dichotomous outcomes, RR and 95\% CI were calculated. When two or more studies presented continuous data from the same validated instrument of evaluation using the same units of measure, data were pooled as a MD with $95 \% \mathrm{CI}$. When primary studies state the same variable using different instruments and different units of measure, we used the standardized mean difference with $95 \% \mathrm{CI}$.

\section{Subgroup analysis and investigation of heterogeneity}

Subgroup analysis for following demographics was planned: age (adolescents, adults and people older than 65); type of surgical intervention TKA; and rehabilitation start (outpatient and inpatient).

\section{Sensitivity analysis}

We planned sensitivity analyses to measure the effects of including trials at risk of selection bias (inadequate or unclear allocation concealment) or detection bias (inadequate or unclear blinding of outcome assessor). We also planned to assess the presence of small study bias (i.e. intervention effect is more beneficial in smaller studies) in the meta-analysis by comparing the fixed- effect estimate with the random-effects estimate for primary outcomes.

\section{'Summary of findings' tables and assessment of the quality of the evidence}

When there is sufficient evidence in future to merit the preparation of summary of findings tables, we will develop these for the main comparisons. We used the GRADE approach to assess the quality of evidence related to each of the key outcomes listed in the types of outcome measures. ${ }^{(17)}$

\section{RESULTS}

\section{Search results}

The search strategy (concluded in August 2013) identified a total of 584 registers at the following current databases: Cochrane Library (129), PubMed (166), Embase (143), LILACS (130), Clinical Trials (11) and Controlled Trials (5).

The search led to the identification of 18 potentially eligible studies, from which the complete papers were retrieved. A total of four studies, published between 1994 and 2013, were included on the review. ${ }^{(18-21)}$

Overall, there were four included studies, eight excluded studies and six ongoing studies (Figure 1).

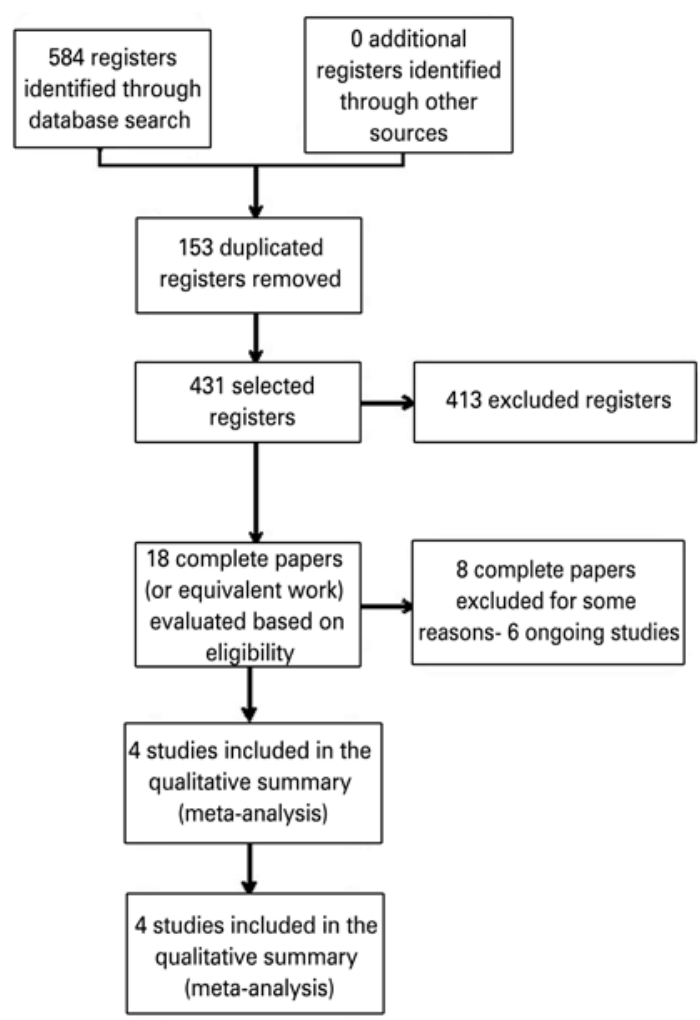

Figure 1. Study flowchart 


\section{Included studies}

This review was based on four randomized clinical trials: Gotlin et al., ${ }^{(18)}$ Levine et al., ${ }^{(19)}$ Petterson et al. ${ }^{(20)}$ and Stevens-Lapsley et al. ${ }^{(21)}$ (all studies presented in English). These trials were found at PubMed, Cochrane Library and Embase. Included studies details are specified at appendix 2 .

\section{Study design}

Gotlin et al., ${ }^{(18)}$ Levine et al., ${ }^{(19)}$ Petterson et al. ${ }^{(20)}$ and Stevens-Lapsley et al. ${ }^{(21)}$ were single-center controlled randomized studies. All studies compared two groups undergoing the same interventions (exercises versus exercises and NMES). Gotlin et al. ${ }^{(18)}$ evaluated exercises and continuous passive motion (Control Group) versus exercises, continuous passive motion and NMES (experimental group).

\section{Participants}

The four studies included trials totalized 376 participants.

\section{Age and sex}

Gotlin et al. ${ }^{(18)}$ described that mean age was 64.8 years in the exercise group (control) and 66.2 years in the NMES plus exercise group (intervention). The Control Group comprised 16 women and 15 men, and the intervention group comprised 20 women and 15 men. In Levine et al., ${ }^{(19)}$ the mean age was 65.1 years in the Control Group, and 68.1 years in the intervention group. The Control Group comprised 21 women and 13 men, and the intervention group was composed of 25 women and 7 men. Petterson et al. ${ }^{(20)}$ reported that the Control Group were composed of 45 woman and 55 were men with mean age of 65.2 years-old. In the intervention group, 47 participants were women and 53 were men, presenting mean age between sexs of 65.3 years-old. In Stevens-Lapsley et al., ${ }^{(21)}$ the sample consisted of 16 women and 15 men in the Control Group and 20 women and 15 men in the intervention group, presenting mean age between sexs of 64.8 years-old for the Control Group and 66.2 years-old for the intervention group.

All participants, in all trials, held a unilateral or bilateral knee osteoarthritis diagnosis. None of the studies described the classification or TKA previous treatment.

\section{Interventions}

The included studies were grouped according to the interventions analyzed.

Gotlin et al. ${ }^{(18)}$ analyzed the effects of exercises and continuous passive motion (Control Group) versus exercises, continuous passive motion and NMES (experimental group) in 40 patients. Levine et al. ${ }^{(19)}$ compared a muscle strengthening program and knee ROM gain versus NMES associated only to knee ROM exercises in 70 participants. Petterson et al. ${ }^{(20)}$ and Stevens-Lapsley et al. ${ }^{(21)}$ compared a progressive muscle (quadriceps) strengthening program versus NMES associated with progressive muscle strengthening, both of early start (immediate postoperative), in 266 participants.

\section{Primary outcomes}

\section{Function or deficiency}

Knee function was evaluated in three studies.(19-21) Levine et al. ${ }^{(19)}$ used Timed Up and Go (TUG), Western Ontario and McMaster Universities Osteoarthritis Index (WOMAC) questionnaire and Knee Society Score (KSS) to measure the joint function. Petterson et al. ${ }^{(20)}$ and Stevens-Lapsley et al. ${ }^{(21)}$ used validated instruments - Timed Up and Go (TUG) Stair Climbing Test (SCT), 6-Minute Walk (6MW) - to evaluate knee functional compromise. Stevens-Lapsley et al. ${ }^{(21)}$ also used the WOMAC questionnaire. In addition to these instruments, Petterson et al. ${ }^{(20)}$ and Stevens-Lapsley et al. ${ }^{(21)}$ evaluated quadriceps muscle strength using dynamometry.

\section{Quality of life}

Gotlin et al. ${ }^{(18)}$ and Levine et al. ${ }^{(19)}$ did not measure this endpoint. Petterson et al. ${ }^{(20)}$ and Stevens-Lapsley et al. ${ }^{(21)}$ evaluated the quality of life using SF-36.

\section{Treatment failure}

All included trials reported no treatment failures.

\section{Secondary outcome \\ Pain}

Pain was evaluated in three studies. ${ }^{(19-21)}$ Levine et al. ${ }^{(19)}$ used the validated score from KSS to measure pain, and Petterson et al. ${ }^{(20)}$ used a validated score (Knee Outcome Survey - KOS and Activities of Daily Living Scale ADLS); Stevens-Lapsley et al. ${ }^{(21)}$ measured individuals pain complaint applying a numeric visual scale. 


\section{Range of motion}

In Gotlin et al., ${ }^{(18)}$ Petterson et al. ${ }^{(20)}$ and Stevens-Lapsley et al. ${ }^{(21)}$ studies were evaluated through goniometry. Levine et al. ${ }^{(19)}$ also evaluated their participants' knee ROM, but did not report which instrument was used to measure this outcome.

\section{Excluded studies}

Eight papers were excluded for not meeting the inclusion criteria. The reasons for exclusion are presented in chart 1.

\section{Ongoing studies}

Our search for ongoing studies resulted in 16 papers on Clinical Trials and Current Controlled Trials. Ten studies were excluded for not meeting our inclusion criteria or being irrelevant. We included: ISRCTN89785408, ISRCTN50117467, NCT01096524， NCT01548040, NCT00224913 and NCT01844193 (Appendix 3).

\section{Risk of bias in the included studies}

All included trials had methodological flaws, rendering them at moderate risk of bias (Figure 2).

\section{Allocation (selection bias)}

Gotlin et al., ${ }^{(18)}$ Levine et al., ${ }^{(19)}$ Petterson et al. ${ }^{(20)}$ and Stevens-Lapsley et al. ${ }^{(21)}$ did not report how the random sequence generation was performed.

Concealment of allocation before assignment was not described in any study, preventing its appreciation (unclear).

\section{Blinding (performance bias and detection bias)}

All trials were judged to be at high risk of performance and detection bias. As they all compared physical therapy interventions, it was not possible to blind treatment providers. No trials included sham intervention; therefore participants were not blinded. It may have

Chart 1. Characteristics of the excluded studies

\begin{tabular}{|lc|}
\hline Study & Reason for exclusion \\
\hline Petterson et al. ${ }^{(22)}$ & Case report \\
Mintken et al. ${ }^{(23)}$ & Case report \\
Stevens et al. ${ }^{(9)}$ & Case series \\
Stevens-Lapsley et al. ${ }^{(24)}$ & Observational study \\
Bade et al. ${ }^{(25)}$ & Literature review \\
Saleh et al. ${ }^{(26)}$ & Literature review \\
Walls et al. ${ }^{(27)}$ & NMES use only in the pre-operative period \\
Stevens et al. ${ }^{(28)}$ & Incomplete unpublished data \\
\hline NMES: neuromuscular electrical stimulation.
\end{tabular}

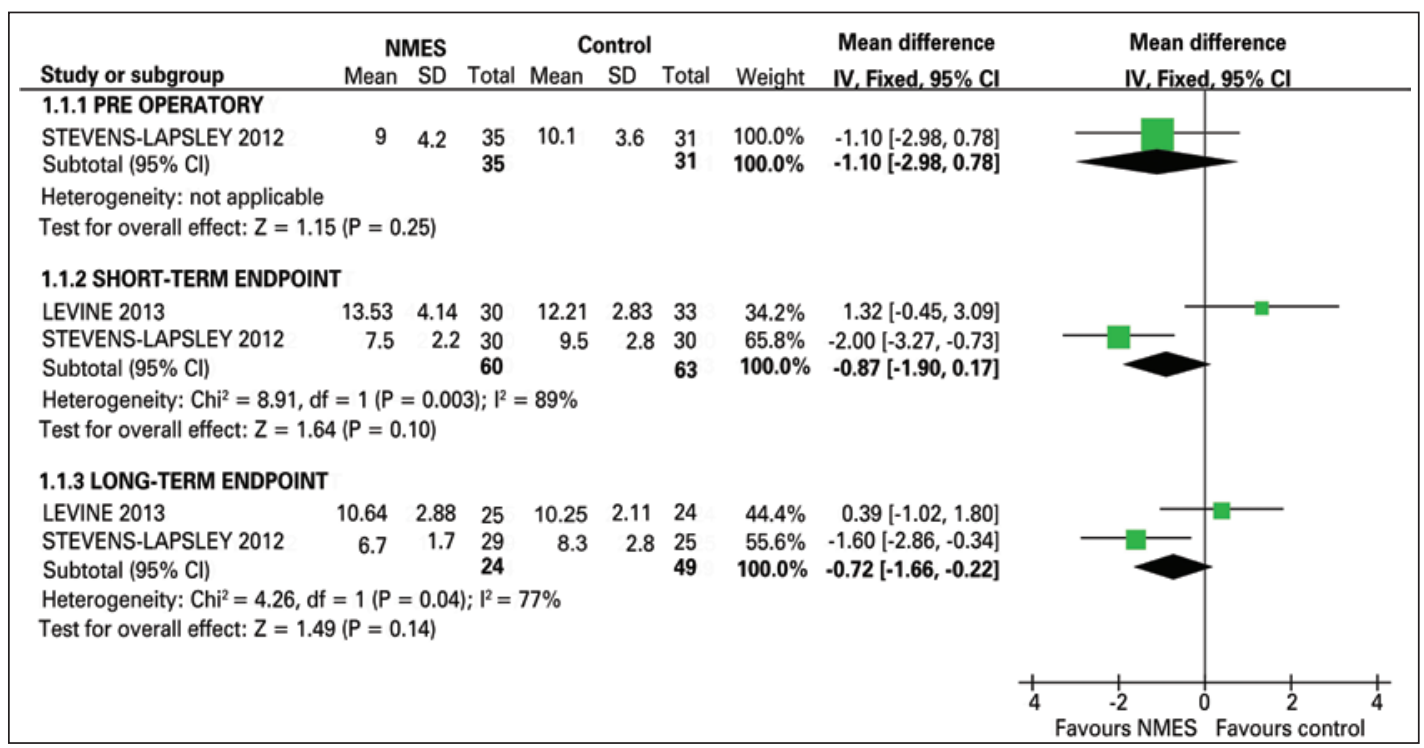

NMES:neuromuscular electrical stimulation. SD: standard deviation; IV:inverse variance; $95 \% \mathrm{Cl}$ : $95 \%$ confidence interval.

Figure 2. Risk of bias chart 
been possible to blind outcome assessors; however, only two trials mentioned assessor blinding. ${ }^{(18-20)}$

\section{Incomplete outcome data (attrition bias)}

Trials with $80 \%$ or more participants completing followup and those whose losses were balanced between intervention groups were deemed as presenting low risk of bias. All included trials ${ }^{(18-21)}$ were considered as presenting low risk of bias.

\section{Selective report (reporting bias)}

Three studies ${ }^{(19-21)}$ were considered as presenting low risk of bias, since their protocols and pre-specified outcomes were available. On the other hand, Gotlin et al. ${ }^{(18)}$ was considered as high risk of bias, since it did not present a specified protocol.

\section{Additional potential bias sources}

Gotlin et al., ${ }^{(18)}$ Petterson et al. ${ }^{(20)}$ and Stevens-Lapsley et al. ${ }^{(21)}$ trials seem to be free from additional biases. Only Levine et al. ${ }^{(19)}$ was considered as high risk of bias, since it not presented a NMES protocol.

\section{Effects of interventions}

The included studies evaluated the following outcomes: function or disability, ROM, quality of life and treatment failure. Additional outcomes planned in our protocol were not evaluated due to insufficient data.

\section{Comparison}

Neuromuscular electrical stimulation versus exercises (with or without continuous passive motion) starting up to the first postoperative week.

\section{Function or disability}

Function measures were analyzed and made available in the following sequence: TUG, $6 \mathrm{MW}$, SCT, quadriceps activation, femoral quadriceps strength, and WOMAC.

\section{Timed Up and Go}

In Levine et al., ${ }^{(19)}$ there were no significant differences in both the short-term (MD: 1.32; 95\% CI: -0.45-3.09) and long-term endpoint (MD: 0.39; 95\% CI: -1.02-1.80). In the Stevens-Lapsley et al. ${ }^{(21)}$ study, we noted a statistically significant difference favoring NMES in both the short-term (MD: -2.00; 95\%CI: -3.27- -0.73) and long-term endpoint (MD: -1.60; 95\%CI: -2.86- -0.34). Studies data grouping did not show any statistically significant difference between the two intervention groups (MD: -0.87; 95\%CI: -1.90-0.17), (MD: -0.72; 95\% CI: -1.66-0.22) at short-term and long-term endpoint, respectively (Figure 3 ).

Petterson et al. ${ }^{(20)}$ reported no statistically significant difference $(p>0.08)$.

\section{6-minute walk test}

Difference between groups in Stevens-Lapsley et al. ${ }^{(21)}$ study marginally favored control at the short-term endpoint (MD: 62.60; 95\%CI: 15.51-109.69). At longterm endpoint, no difference between groups was noted (MD: 46.80; 95\%CI: -0.53-94.13). No statistically significant difference was reported by Petterson et al. ${ }^{(20)}$ $(p>0.08)$.

\section{Stair Climbing Test}

Petterson et al. ${ }^{(20)}$ report no significant difference for this outcome at the short-term or long-term endpoint $(\mathrm{p}>0.08)$. At Stevens-Lapsley et al. ${ }^{(21)}$, no statistically significant difference between the two groups NMES and exercises (MD: -2.70; 95\%CI: -6.40-1.00), (MD: -3.30 ; 95\%CI: -7.27-0.67) was noted in both short-term and long-term endpoint, respectively.

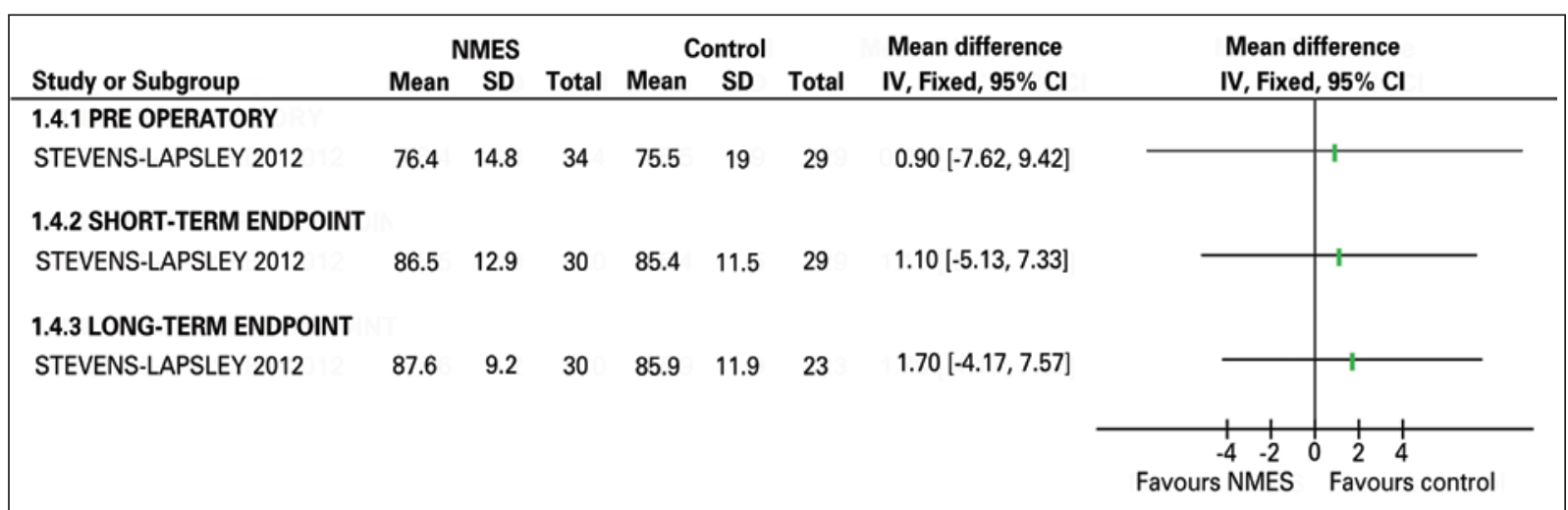

NMES: neuromuscular electrical stimulation. SD: standard deviation; IV: inverse variance; $95 \% \mathrm{Cl}: 95 \%$ confidence interval.

Figure 3. Timed Up and Go graph 


\section{Quadriceps activation (\%)}

Petterson et al. ${ }^{(20)}$ reported the same, with $\mathrm{p}>0.08$. Stevens-Lapsley et al. ${ }^{(21)}$ reported no statistically significant difference at the short-term endpoint (MD: 1.10; 95\%CI: -5.13-7.33) between both groups and at the post-TKA short-term endpoint (MD: 1.70; 95\%CI: -4.17-7.57) (Figure 4).

\section{Femoral quadriceps strength $(\mathbf{N}-\mathbf{m} / \mathbf{k g})$}

Petterson et al. ${ }^{(20)}$ also reported the lack of significant difference between groups $(p>0.08)$. No statistically significant differences between the two groups at the short-term (MD: 0.22; 95\% CI: -0.02-0.46) and the longterm endpoint (MD: 0.16; 95\% CI: -0.09-0.41) were noted at Stevens-Lapsley et al. ${ }^{(21)}$

\section{Quality of life}

The SF-36 questionnaire was used to measure the quality of life during and after the physical therapy intervention in both Petterson et al. ${ }^{(20)}$ and Stevens-Lapsley et al. ${ }^{(21)}$ studies and data were analyzed according to its components.

\section{SF-36 physical component summary}

Petterson et al. ${ }^{(20)}$ reported no significant difference between groups $(p>0.08)$. Stevens-Lapsley et al. ${ }^{(21)}$ also reported no statistically significant differences at the short-term (MD: 3.20; 95\% CI: -1.50-7.90) and the longterm endpoint (MD: 1.90; 95\%CI: -1.97-5.77).

\section{SF-36 mental component summary}

Petterson et al. ${ }^{(20)}$ reported no significant difference, with $\mathrm{p}>0.08$. At the mental component summary, Stevens-Lapsley et al. ${ }^{(21)}$ also reported no statistically significant difference in both the short-term (MD: 3.70; 95\% CI: -1.55 -8.95) and long-term endpoint (MD: 3.00; $95 \%$ CI: $-0.39-6.39)$.

\section{Quality of life and function}

WOMAC questionnaire was used by Levine et al. ${ }^{(19)}$ to measure knee function, with no statistically significant difference between groups in both the short-term (MD:5.67;95\% CI:-1.88-13.22) and long-term endpoint (MD: 5.81; 95\%CI: -3.01-14.63). Stevens-Lapsley et al. ${ }^{(21)}$ also reported no statistically significant difference in both the short-term (MD: -6.80; 95\%CI: -15.04-1.44) and at the long-term endpoint (MD: -4.30; 95\% CI: -9.99-1.39). Data pooling showed no statistically significant difference in both the short-term (MD: -0.02; 95\%CI: -5.59-5.54) and at the long-term endpoint (MD: -1.33; 95\%CI: -6.11 -3.45 ) between the two intervention groups.

Levine et al. ${ }^{(19)}$ used the KSS questionnaire to check knee function and reported no statistically significant difference in both the short-term (MD: 4.97; 95\% CI: -4.65-14.59) and the long-term endpoint (MD: 7.92; 95\% CI: -2.29-18.13).

Petterson et al. ${ }^{(20)}$ used the KOS questionnaire to measure knee function and also reported no statistically significant difference in both short-term and long-term endpoint $(p>0.01)$.

\section{Treatment failure}

All included trials reported no treatment failures.

\section{Pain}

None of the included studies individually evaluated this outcome.

\section{Range of motion \\ Knee flexion}

Levine et al. ${ }^{(19)}$ reported no statistically significant difference at short-term (MD: -3.20 ; 95\% CI: -8.52 -2.12) and long-term endpoint (MD: 2.30; 95\%CI: -4.25

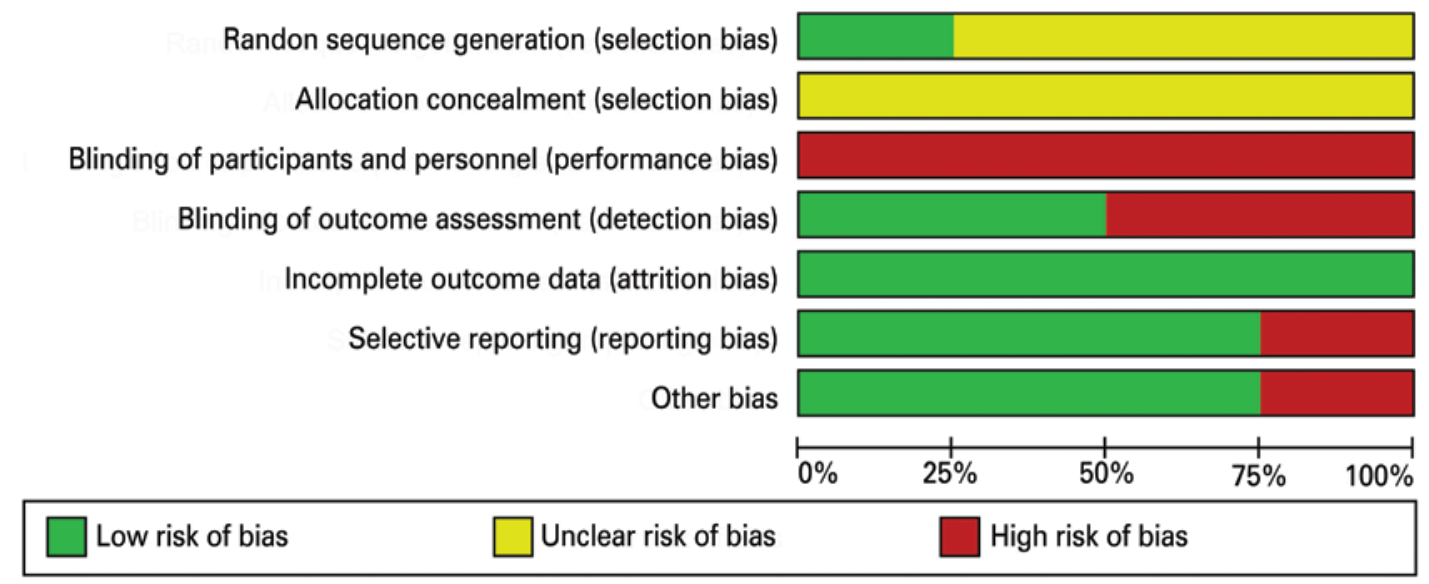

Figure 4. Quadriceps activation chart 
-8.85). Stevens-Lapsley et al. ${ }^{(21)}$ reported no statistically significant difference between groups - NMES and exercises (MD: 2.90; 95\%CI: -1.89-7.69 and MD: 2.40; 95\% CI: -1.82-6.62) at the short-term and long-term endpoint, respectively. Petterson et al. ${ }^{(20)}$ also reported no statistically significant difference $(\mathrm{p}>0.01)$. Data pooling showed no statistically significant difference between the two intervention groups (MD: $0.17 ; 95 \% \mathrm{CI}$ : -3.39-3.73; and MD: 2.37; 95\%CI: -1.18-5.92) in both the short-term and long-term endpoint, respectively.

\section{Knee extension}

Gotlin et al. ${ }^{(18)}$ and Stevens-Lapsley et al. ${ }^{(21)}$ reported a statistically significant difference favoring the intervention group compared with the group without intervention in the short-term endpoint (MD: -2.65; 95\% CI: -4.05- -1.25 versus $\mathrm{MD}$ : -2.40 ; 95\% CI -4.09- -0.71) respectively, with no statistically significant difference in the longterm endpoint, that was reported only in the study by Stevens-Lapsley et al. ${ }^{(21)}$ (MD: -0.60; 95\%CI: -2.43-1.23). Levine et al. ${ }^{(19)}$ reported no significant difference in both the short-term endpoint (MD: 0.65; 95\% CI -2.00 -3.30) and the long-term endpoint (MD: -0.96 ; 95\% CI: $-3.62-1.70)$. When data were pooled, a statistically significant difference in the short-term endpoint was noted (MD: -2.09; 95\%CI: -3.09- -1.09); however, there was no statistically significant difference in the longterm endpoint (MD: -0.72; 95\%CI: -2.22-0.79) in both intervention groups.

Petterson et al. ${ }^{(20)}$ report no significant difference between both groups during all months of intervention $(\mathrm{p}>0.01)$.

\section{DISCUSSION}

At this review, only randomized clinical trials were included, leading to the analysis of four trials deemed of moderate risk of bias and evaluating kinesiotherapic physical therapy interventions compared to NMES with physiotherapy use in 376 participants undergoing TKA.

No evidence indicated if NMES with physiotherapy provided benefits regarding the quality of life. The postoperative treatment with NMES can improve the femoral quadriceps function, but we are not sure about the effectiveness of this intervention, due to the low quality evidence.

Very low evidence from the included trials presented a low general quality resulting from methodological failures, including the lack of allocation concealment and participants and personnel blinding in all trials. However, the quantitative results of this review must be carefully interpreted, requiring confirmation of such data by evidence derived from high methodological quality trials.

We believe that our search strategy was complete, with no language restriction. However, it is possible that we missed some potentially eligible studies. We tried to contact the included studies' authors in order to obtain some data, but with no success.

We found a systematic review comparing NMES versus exercise therapy to treat the quadriceps inhibition after TKA (Monaghan et al). ${ }^{(29)}$ including two randomized and non-randomized clinical trials, and a total of 69 subjects. Our study results are consistent with the Cochrane systematic review results and our conclusion is similar to this publication.

Monaghan et al. ${ }^{(29)}$ evaluated NMES use for quadriceps strengthening pre-and post-TKA and reported no significant differences for these outcomes; however, they considered included studies with a high risk of bias due to study design limitations and presented results imprecision, preventing a meta-analysis performance. The authors concluded that the identified studies do not allow any definition regarding NMES pre- or postTKA.

Monaghan et al. ${ }^{(29)}$ also interpreted that participants undergoing quadriceps NMES presented a slight advantage in function improvement and less deficiency than those conservatively treated in the short follow-up, just as we see at the clinical practice and consistent with the findings in Gotlin et al. ${ }^{(18)}$ However, efficacy was reduced at the long-term follow-up.

Therefore, this review is inconclusive about NMES efficacy, and further evidence is required to support or deny its use at quadriceps activation after TKA. The authors are aware that this review subject is the object of an ongoing investigation and could be updated in order to incorporate new evidences.

\section{CONCLUSION}

The very low evidences from included studies found on this review do not allow any conclusions regarding neuromuscular stimulation application for quadriceps strengthening with physical therapy before or after total knee replacement. Until now, evidence for neuromuscular stimulation use to quadriceps strengthening in this patient group is unclear. However, it is critical that future studies verify the quadriceps strength pre- and post-neuromuscular stimulation using reliable evaluations and validated tools, as well as a clear description of the applied dose in the study design. It is also of uttermost importance that results be presented appropriately for meta-analysis performance. 


\section{ACKNOWLEDGEMENTS}

The authors would like to thank Renata Scavone de Oliveira who kindly contributed revising the original text in English.

\section{REFERENCES}

1. Pereira D, Peleteiro B, Araújo J, Branco J, Santos RA, Ramos E. The effect of osteoarthritis definition on prevalence and incidence estimates: a systematic review. Osteoarthritis Cartilage. 2011;19(11):1270-85. Review.

2. Labraca NS, Castro-Sánchez AM, Matarán-Peñarrocha GA, Arroyo-Morales M, Sánchez-Joya Mdel M, Moreno-Lorenzo C. Benefits of starting rehabilitation within 24 hours of primary total knee arthroplasty: randomized clinical trial. Clin Rehabil. 2011;25(6):557-66.

3. Rosal MC, Ayers D, Li W, Oatis C, Borg A, Zheng H, et al. A randomized clinical trial of a peri-operative behavioral intervention to improve physical activity adherence and functional outcomes following total knee replacement. BMC Musculoskelet Disord. 2011;12:226.

4. Piva SR, Gil AB, Almeida GJ, DiGioia AM 3rd, Levison TJ, Fitzgerald GK. A balance exercise program appears to improve function for patients with total knee arthroplasty: a randomized clinical trial. Phys Ther. 2010;90(6):880-94 .

5. Moffet H, Collet JP, Shapiro SH, Paradis G, Marquis F, Roy L. Effectiveness of intensive rehabilitation on functional ability and quality of life after first total knee arthroplasty: a single-blind randomized controlled trial. Arch Phys Med Rehabil. 2004;85(4):546-56.

6. Ahmed AR, Abd-Elkader SM, Al-Obathani KS. Effect of a 6-week rehabilitation program on gait parameters after total knee arthroplasty. Saudi Med J. 2010;31(9):1032-5.

7. Snyder-Mackler L, Delitto A, Bailey SL, Stralka SW. Strength of the quadriceps femoris muscle and functional recovery after reconstruction of the anterior cruciate ligament. A prospective, randomized clinical trial of electrical stimulation. J Bone Joint Surg Am. 1995;77(8):1166-73.

8. Rosenberg TD, Franklin JL, Baldwin GN, Nelson KA. Extensor mechanism function after patellar tendon graft harvest for anterior cruciate ligament reconstruction. Am J Sports Med. 1992;20(5):519-25; discussion 525-6.

9. Stevens JE, Mizner RL, Snyder-Mackler L. Neuromuscular electrical stimulation for quadriceps muscle strengthening after bilateral total knee arthroplasty: a case series. J Orthop Sports Phys Ther. 2004;34(1):21-9.

10. Snyder-Mackler L, De Luca PF, Williams PR, Eastlack ME, Bartolozzi AR 3rd. Reflex inhibition of the quadriceps femoris muscle after injury or reconstruction of the anterior cruciate ligament. J Bone Joint Surg Am. 1994;76(4):555-60.

11. Lysholm J, Gillquist J. Evaluation of knee ligament surgery results with special emphasis on use of a scoring scale. Am J Sports Med. 1982;10(3):150-4.

12. Ware JE Jr, Sherbourne CD. The MOS 36-item short-form health survey (SF-36). I. Conceptual framework and item selection. Med Care. 1992;30(6):473-83.

13. Lefebvre $C$, Manheimer $E$, Glanville $J$ on behalf of the Cochrane Bias Methods Group. Searching for studies. In: Higgins JP, Green S, editors. Cochrane Handbook for Systematic Reviews of Interventions. Version 5.1.0 [update Marc 2011]. [Internet] London (UK): The Cochrane Collaboration; 2011. [cited 2015 Aug 5]. Available from: http://handbook.cochrane.org/ chapter_6/6_searching_for_studies.htm

14. Higgins JP, Deeks JJ, Altman DG on behalf of the Cochrane Bias Methods Group. Special topics in statistics. In: Higgins JP, Green S, editors. Cochrane Handbook for Systematic Reviews of Interventions. Version 5.1.0 [update Marc 2011]. [Internet] London (UK): The Cochrane Collaboration; 2011 [cited 2015 Aug 5]. Available from: http://handbook.cochrane.org/
15. Deeks JJ, Higgins JP, Altman DG, editors, on behalf of the Cochrane Bias Methods Group. Analysing data and undertaking meta-analyses. In: Higgins JP, Green S, editors. Cochrane Handook for Systematic Reviews of Interventions. Version 5.1.0 [update Marc 2011]. [Internet] London (UK): The Cochrane Collaboration; 2008 [cited 2015 Aug 5]. Available from: http://handbook.cochrane.org/

16. Sterne JA, Egger M, Moher D on behalf of the Cochrane Bias Methods Group. Addressing reporting biases. In: Higgins JP, Green S, editors. Cochrane Handook for systematic Reviews of Intervention. Version 5.1.0 [update Marc 2011]. [Internet] London (UK): The Cochrane Collaboration; 2008 [cited 2015 Aug 5]. Available from: http://handbook.cochrane.org/chapter 10/10 addressing_reporting_biases.htm

17. Schünemann HJ, Oxman $A D$, Vist GE, Higgins JP, Deeks JJ, Glasziou P, and Gordon H Guyatt on behalf of the Cochrane Applicability and Recommendations Methods Group. Interpreting results and drawing conclusions. In: Higgins JP, Green S, editors. Cochrane Handbook for Systematic Reviews of Interventions. Version 5.1.0 [updated Marc 2011]. [Internet] London (UK): The Cochrane Collaboration; 2011 [cited 2015 Aug 5]. Available from: http://handbook.cochrane.org/ chapter 12/12 interpreting results and drawing conclusions.htm

18. Gotlin RS, Hershkowitz S, Juris PM, Gonzalez EG, Scott WN, Insall JN Electrical stimulation effect on extensor lag and length of hospital stay after total knee arthroplasty. Arch Phys Med Rehabil. 1994;75(9):957-9.

19. Levine M, McElroy K, Stakich V, Cicco J. Comparing conventional physical therapy rehabilitation with neuromuscular electrical stimulation after TKA. Orthopedics. 2013;36(3):e319-24.

20. Petterson SC, Mizner RL, Stevens JE, Raisis L, Bodenstab A, Newcomb W, et al. Improved function from progressive strengthening interventions after total knee arthroplasty: a randomized clinical trial with an imbedded prospective cohort. Arthritis Rheum. 2009;61(2):174-83

21. Stevens-Lapsley JE, Balter JE, Wolfe P, Eckhoff DG, Kohrt WM. Early neuromuscular electrical stimulation to improve quadriceps muscle strength after total knee arthroplasty: a randomized controlled trial. Phys Ther. 2012; 92(2):210-26.

22. Petterson S, Snyder-Mackler L. The use of neuromuscular electrical stimulation to improve activation deficits in a patient with chronic quadriceps strength impairments following total knee arthroplasty. J Orthop Sports Phys Ther. 2006;36(9):678-85.

23. Mintken PE, Carpenter KJ, Eckhoff D, Kohrt WM, Stevens JE. Early neuromuscular electrical stimulation to optimize quadriceps muscle function following total knee arthroplasty: a case report. J Orthop Sports Phys Ther. 2007;37(7):364-71.

24. Stevens-Lapsley JE, Balter JE, Wolfe P, Eckhoff DG, Schwartz RS, Schenkman $\mathrm{M}$, et al. Relationship between intensity of quadriceps muscle neuromuscular electrical stimulation and strength recovery after total knee arthroplasty. Phys Ther. 2012;92(9):1187-96

25. Bade MJ, Stevens-Lapsley JE. Restoration of physical function in patients following total knee arthroplasty: an update on rehabilitation practices. Curr Opin Rheumatol. 2012;24(2):208-14. Review.

26. Saleh KJ, Lee LW, Gandhi R, Ingersoll CD, Mahomed NN, Sheibani-Rad S, et al. Quadriceps strength in relation to total knee arthroplasty outcomes. Instr Course Lect. 2010;59:119-30. Review.

27. Walls RJ, McHugh G, O'Gorman DJ, Moyna NM, O'Byrne JM. Effects of preoperative neuromuscular electrical stimulation on quadriceps strength and functional recovery in total knee arthroplasty. A pilot study. BMC Musculoskelet Disord. 2010;11:119.

28. Stevens J. Physiologically-based rehabilitation for patients after total knee arthroplasty. USA: University of Delaware; 2002.

29. Monaghan B, Caulfield B, O'Mathúna DP. Surface neuromuscular electrical stimulation for quadriceps strengtheing pre and post total knee replacement. Cochrane Database Syst Rev. 2010;20(1):CD007177. Review. 
Appendix 1. Search strategy

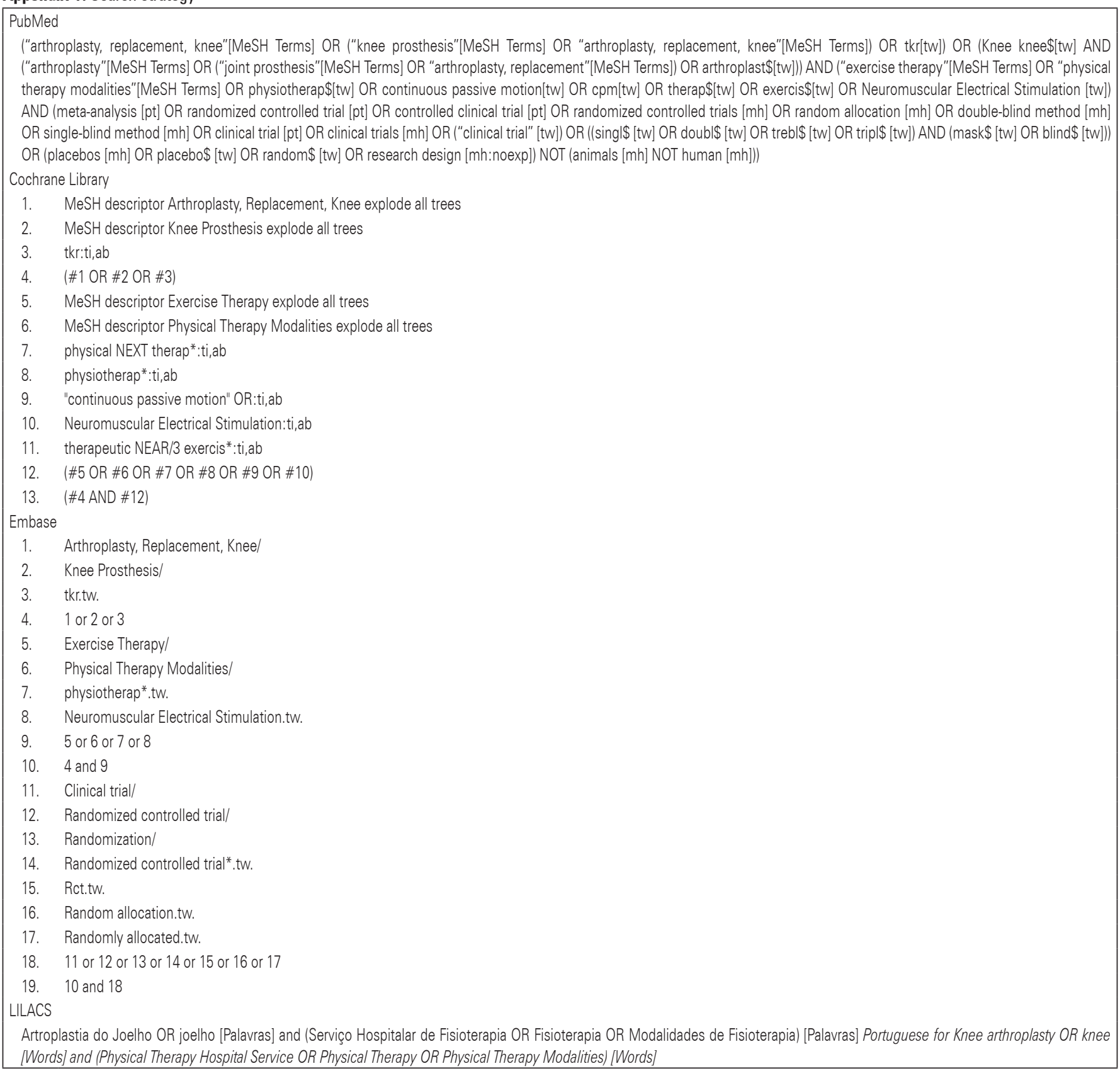


Appendix 2. Extraction form of the characteristics of the included studies

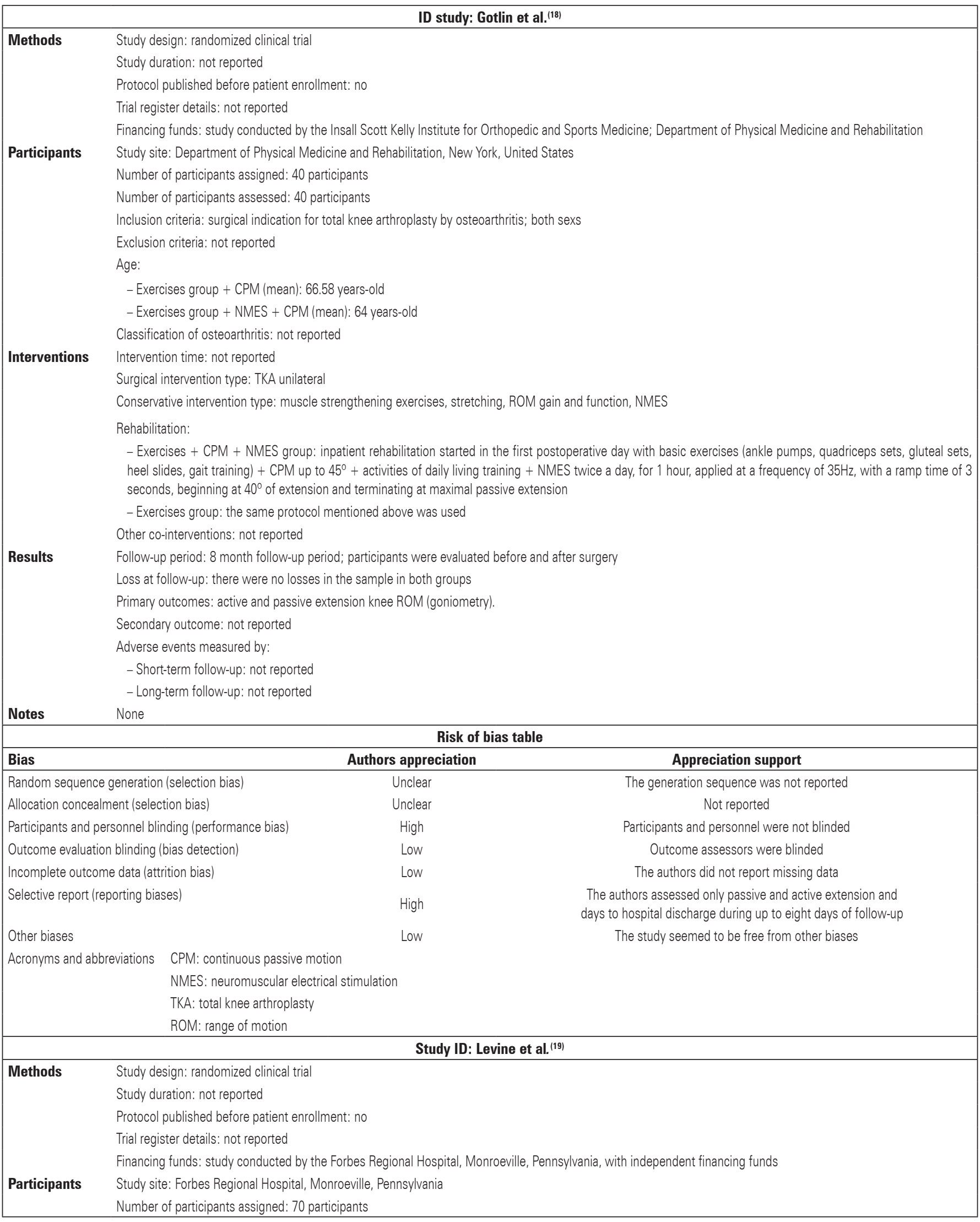


...Continuation

Appendix 2. Extraction form of the characteristics of the included studies

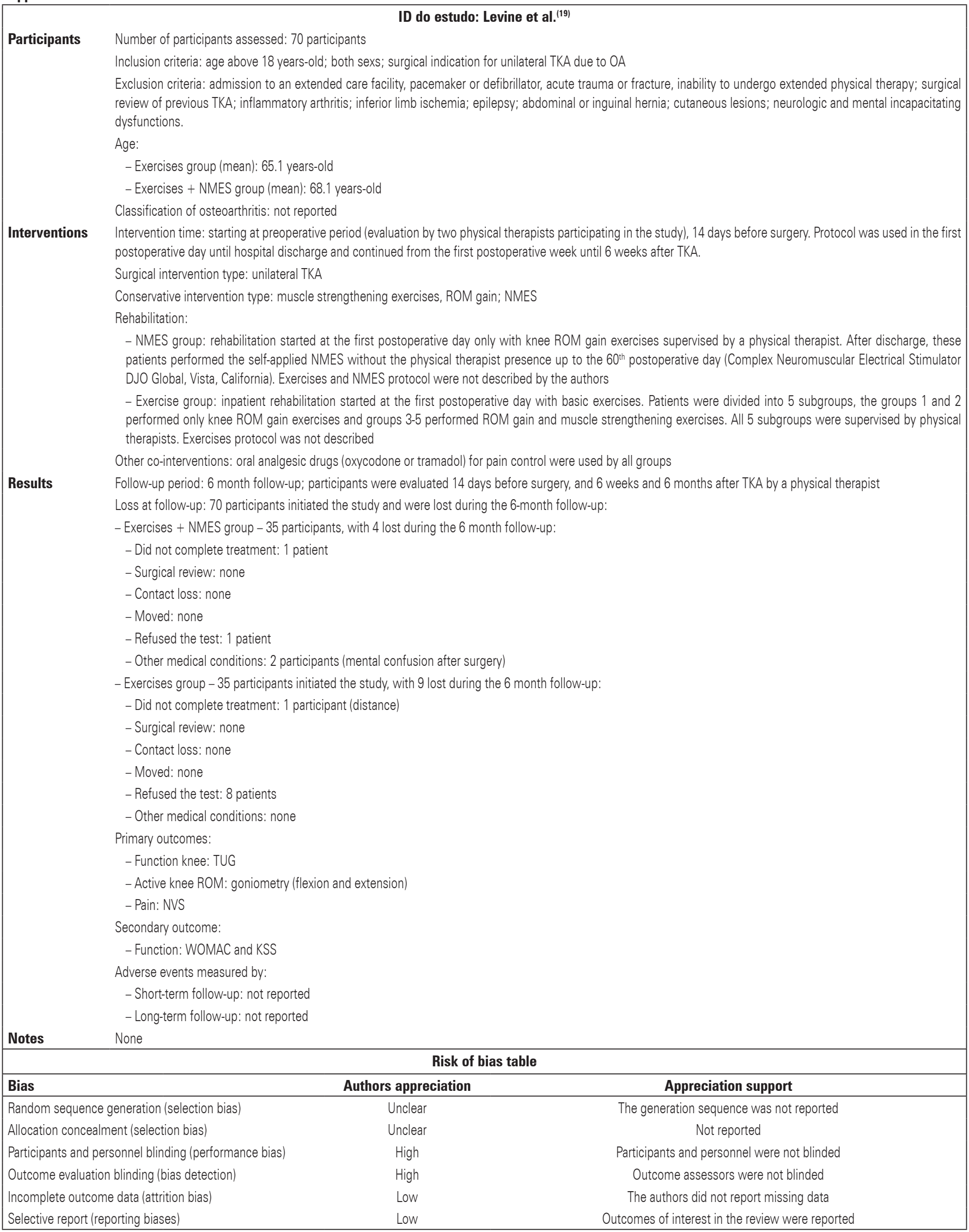


.... Continuation

Appendix 2. Extraction form of the characteristics of the included studies

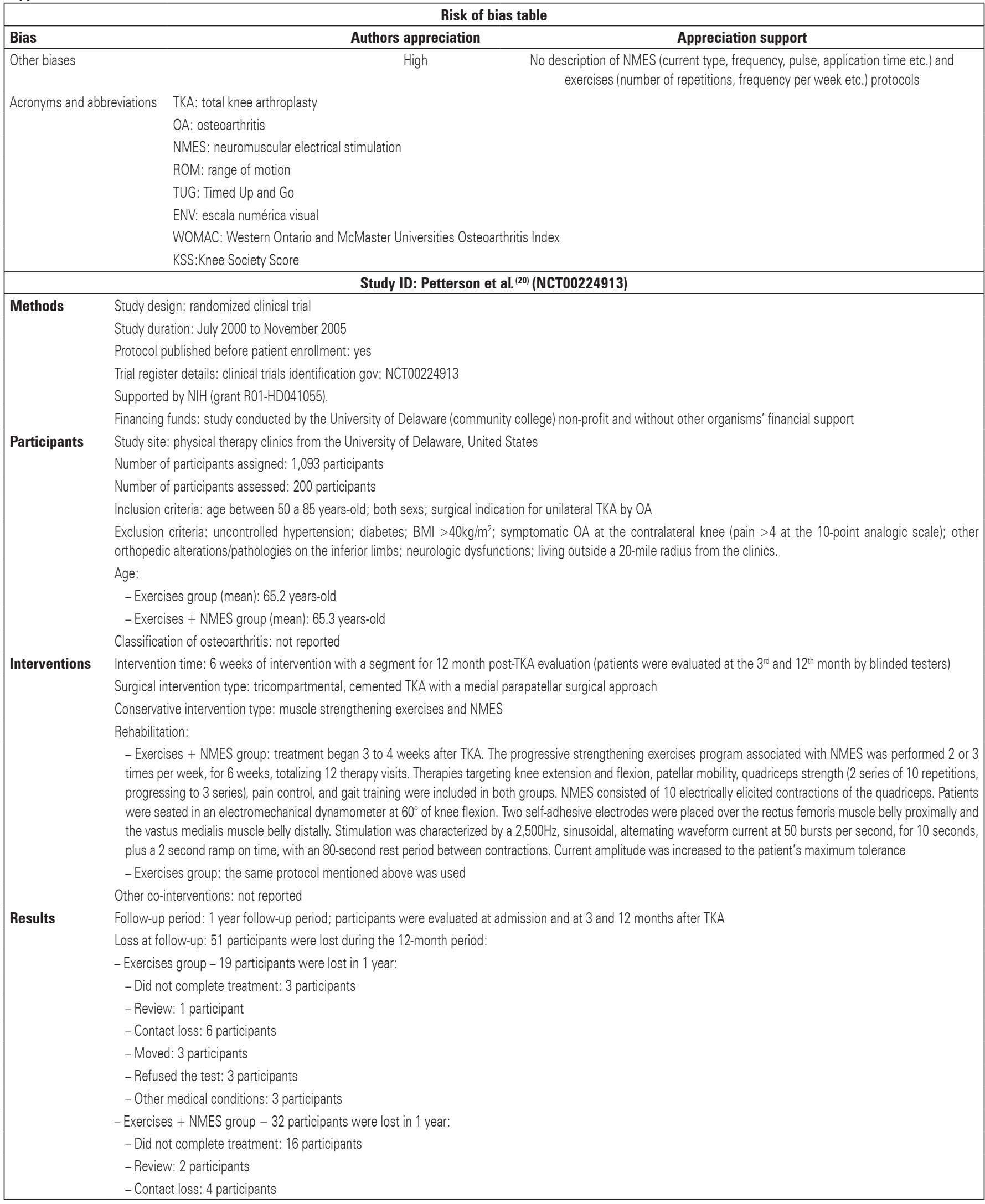


...Continuation

Appendix 2. Extraction form of the characteristics of the included studies

\begin{tabular}{|c|c|c|c|}
\hline \multicolumn{4}{|c|}{ ID do estudo: Petterson et al. ${ }^{(20)}$ (NCT00224913) } \\
\hline \multirow[t]{13}{*}{ Results } & \multicolumn{3}{|c|}{ - Moved: none } \\
\hline & \multicolumn{3}{|c|}{ - Refused the test: 3 participants } \\
\hline & \multicolumn{3}{|c|}{ - Other medical conditions: 7 participants } \\
\hline & \multicolumn{3}{|c|}{ Primary outcomes: } \\
\hline & \multicolumn{3}{|c|}{ - Quadriceps muscle strength: dynamometry (isokinetic) } \\
\hline & \multicolumn{3}{|c|}{ - Function knee: TUG, SCT, and 6MW } \\
\hline & \multicolumn{3}{|c|}{ - Active knee ROM: goniometry. } \\
\hline & \multicolumn{3}{|c|}{ Secondary outcome: } \\
\hline & \multicolumn{3}{|c|}{ - Quality of life: SF-36 and KOS, ADLS } \\
\hline & \multicolumn{3}{|c|}{ - Pain: KOS, ADLS } \\
\hline & \multicolumn{3}{|c|}{ Adverse events measured by: } \\
\hline & \multicolumn{3}{|c|}{ - Short-term follow-up: not reported } \\
\hline & \multicolumn{2}{|c|}{ - Long-term follow-up: not reported } & \\
\hline Notes & \multicolumn{3}{|c|}{ We contacted the authors by e-mail more than once in order to obtain the missing data, but with no response so far } \\
\hline \multicolumn{4}{|c|}{ Risk of bias table } \\
\hline \multicolumn{3}{|r|}{ Authors appreciation } & Appreciation support \\
\hline \multicolumn{3}{|c|}{ Random sequence generation (selection bias) } & The generation sequence was not reported \\
\hline \multicolumn{3}{|c|}{ Allocation concealment (selection bias) } & The concealment method was not reported \\
\hline \multicolumn{3}{|c|}{ Participants and personnel blinding (performance bias) } & Participants and personnel were not blinded \\
\hline \multicolumn{3}{|c|}{ Outcome evaluation blinding (bias detection) } & Outcome assessors were blinded \\
\hline Incomplete out & ome data (attr & rition bias) Low & $\begin{array}{l}\text { Outcome data figure-balanced between intervention groups, with similar } \\
\text { reasons for lack of data between groups }\end{array}$ \\
\hline Selective repor & (reporting bias & Low & $\begin{array}{l}\text { The study protocol was available and all of the study's pre-specified } \\
\text { (primary and secondary) outcomes that are of interest for this review } \\
\text { have been reported in the pre-specified way }\end{array}$ \\
\hline Other biases & & Low & The study seems to be free from other biases \\
\hline Acronyms and & bbreviations & NIH: National Institutes of Health & \\
\hline & & TKA: total knee arthroplasty & \\
\hline & & OA: osteoarthritis & \\
\hline & & BMl: body mass index & \\
\hline & & NMES: neuromuscular electrical stimulation & \\
\hline & & ROM: range of motion & \\
\hline & & TUG: Timed Up and Go & \\
\hline & & SCT: Stair Climbing Test & \\
\hline & & 6MW: 6-minute Walk & \\
\hline & & SF-36: Short Form Health Survey-36 & \\
\hline & & KOS ADLS KSS: Knee Outcome Survey Activities of Daily Liv & \\
\hline & & ID study: Stevens-Laps & (NCT00800254) \\
\hline Methods & Study desig & n: randomized clinical trial & \\
\hline & Study durati & ion: June 2006 to June 2010 & \\
\hline & Protocol puk & blished before patient enrollment: yes. & \\
\hline & Trial register & details: ClinicalTrials.gov Identifier: NCT00800254 & \\
\hline & $\begin{array}{l}\text { Financing fu } \\
\text { Rheumatolo }\end{array}$ & $\begin{array}{l}\text { unds: study conducted by the University of Colorado Hospit } \\
\text { gy }\end{array}$ & ed by the National Institute of Aging (K23AG029978) and the American College of \\
\hline Participants & Study site: & University of Colorado Physical Therapy Clinics, United States & \\
\hline & Number of $p$ & participants assigned: 526 participants. & \\
\hline & Number of $p$ & participants assessed: 66 participants. & \\
\hline & Inclusion cri & iteria: age between 50 and 85 years-old; both sexs; surgical in & unilateral TKA by $O A$ \\
\hline & $\begin{array}{l}\text { Exclusion } \mathrm{cr} \\
\text { other orthop }\end{array}$ & $\begin{array}{l}\text { riteria: uncontrolled hypertension; uncontrolled diabetes; BM } \\
\text { oedic alterations/pathologies on the inferior limbs; incapacitati }\end{array}$ & $\begin{array}{l}\text { symptomatic } O A \text { at the contralateral knee (pain }>4 \text { at the 10-point analogic scale); } \\
\text { ic alterations }\end{array}$ \\
\hline & Age: & & \\
\hline & - Exercise & group (mean): 65.2 years-old & \\
\hline & - Exercise & es group + NMES (mean): 65.3 years-old & \\
\hline & Lesion class & sification: not reported & \\
\hline
\end{tabular}


.... Continuation

Appendix 2. Extraction form of the characteristics of the included studies

\section{ID do estudo: Stevens-Lapsley et al. ${ }^{(21)}$ (NCT00800254)}

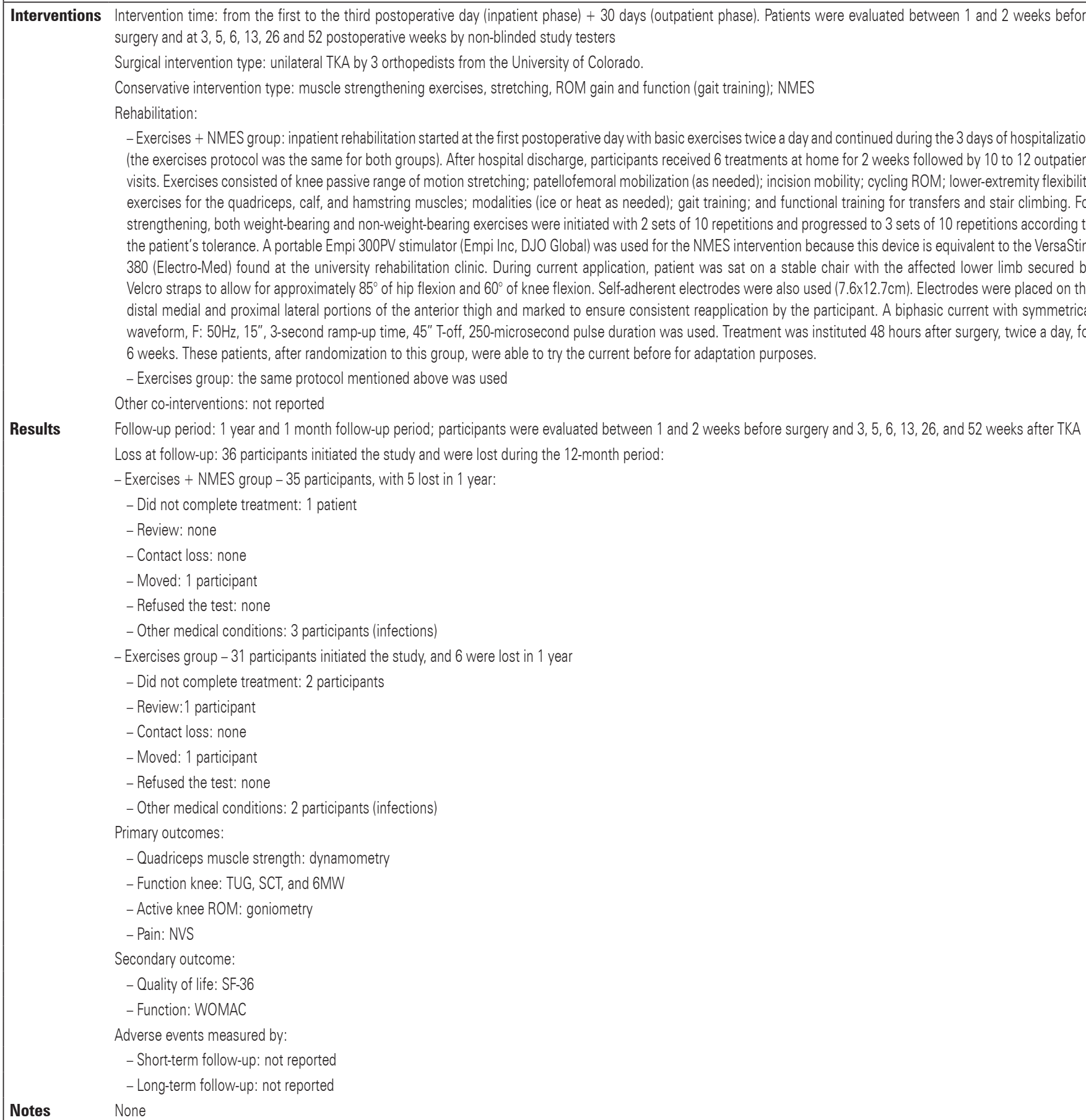

Notes None

\begin{tabular}{|lcc|}
\hline Bias & Authors appreciation & Appreciation support \\
\hline Random sequence generation (selection bias) & Low & Blocked randomization was described with random block sizes of 4, 6, or 8 \\
Allocation concealment (selection bias) & Unclear & The concealment method was not reported \\
Participants and personnel blinding (performance bias) & High & Participants and personnel were not blinded \\
Outcome evaluation blinding (bias detection) & High & Outcome assessors were not blinded \\
Incomplete outcome data (attrition bias) & Low & Outcome data figure-balanced between intervention groups, with similar reasons \\
& & for lack of data between groups \\
\hline
\end{tabular}


.... Continuation

Appendix 2. Extraction form of the characteristics of the included studies

\begin{tabular}{|c|c|c|c|}
\hline \multicolumn{4}{|c|}{ Risk of bias table } \\
\hline \multicolumn{2}{|l|}{ Bias } & Authors appreciation & Appreciation support \\
\hline \multicolumn{2}{|c|}{ Selective report (reporting biases) } & Low & $\begin{array}{l}\text { The study protocol was available and all of the study's pre-specified (primary and secondary) } \\
\text { outcomes that are of interest for this review have been reported in the prespecified way }\end{array}$ \\
\hline \multirow{12}{*}{$\begin{array}{l}\text { Other biases } \\
\text { Acronyms and } \\
\text { abbreviations }\end{array}$} & & Low & The study seems to be free from other biases. \\
\hline & TKA: & & \\
\hline & OA: 0 & & \\
\hline & BMl: & & \\
\hline & $\mathrm{ROM}$ & & \\
\hline & NMES & imulation & \\
\hline & PRON & & \\
\hline & TUG: & & \\
\hline & SCT: & & \\
\hline & $6 \mathrm{MW}$ & & \\
\hline & SF-36 & & \\
\hline & WOM & Master Universities Osteoa & nritis Index \\
\hline
\end{tabular}

Appendix 3. Ongoing studies characteristics

\begin{tabular}{|c|c|}
\hline \multicolumn{2}{|r|}{ ISRCTN89785408 } \\
\hline Title & Kneehab ${ }^{\mathrm{TM}}$ pre and post total knee replacement surgery \\
\hline \multirow[t]{4}{*}{ Methods } & Study design: randomized controlled blinded parallel group trial \\
\hline & Random sequence generation: not reported \\
\hline & Allocation concealment: not reported \\
\hline & Masking: not reported \\
\hline \multirow[t]{4}{*}{ Participants } & Location: Liverpool, United Kingdom \\
\hline & Target sample size (n): 200 participants \\
\hline & $\begin{array}{l}\text { Inclusion criteria: individuals who are scheduled for total knee replacement surgery; individuals who are at least } 18 \text { years of age; individuals with a BMl <35; } \\
\text { individuals who are walking independently with or without assistive devices; must be able and willing to complete all study, assessments and to be followed } \\
\text { for the full course of the study; must be able to read, write and follow instructions in English; must be able and willing to provide informed consent; must } \\
\text { be willing and able to attend the additional preoperative assessment }\end{array}$ \\
\hline & $\begin{array}{l}\text { Exclusion criteria: individuals who have failed the pre-TKA operative assessment; individuals with a history of foot and/or ankle pathology; individuals } \\
\text { with a history of tibial or femoral fractures; individuals with a history of any underlying neurological conditions; individuals with physical conditions which } \\
\text { would make them unable to perform study procedures; individuals with a total hip replacement; individuals undergoing revision TKA of the same operated } \\
\text { leg; pregnant women or inadequate precautions to prevent pregnancy; diagnosis of a medical condition that would contraindicate treatment with the } \\
\text { product, e.g. skin lesions at electrode site; individuals with an active implanted medical device (i.e. pacemaker, pump); individuals with a history of stroke; } \\
\text { individuals with a history of neurological disorder that affects lower extremity function (stroke, peripheral neuropathy, Parkinson's disease, multiple sclerosis } \\
\text { etc.); individuals with a diagnosis of inflammatory arthritis (rheumatoid arthritis, gout or psoriatic arthritis); individuals with muscle diseases (i.e. muscular } \\
\text { dystrophy); visible skin injury or disease on their legs; principal investigator for this study, or member of study staff }\end{array}$ \\
\hline \multirow[t]{2}{*}{ Interventions } & Kneehab ${ }^{\mathrm{TM}}$ group: 6 weeks before and 6 weeks TKA (30 minutes of NMES twice per day for 12 weeks), plus standard physiotherapy \\
\hline & Control group: 12 weeks (6 weeks pre and post) standard physiotherapy \\
\hline \multirow[t]{6}{*}{ Results } & Primary outcomes: \\
\hline & Determine the efficacy of Kneehab ${ }^{\top M}$ in promoting early recovery of quadriceps performance in patients recovering from TKA as determined by: \\
\hline & - Clinically significant increase in isometric extensor strength compared to controls \\
\hline & - Clinically significant reduction in TUG and SCT score compared to controls \\
\hline & Secondary outcomes: quality of life measures; health economic outcomes \\
\hline & Timing of outcomes measurement: not reported \\
\hline \multirow[t]{5}{*}{ Starting date } & Main ID: ISRCTN89785408 \\
\hline & Anticipate start date: April 12, 2010 \\
\hline & Anticipate last date: July 1, 2011 \\
\hline & Status: not recruiting. \\
\hline & Estimated study completion date: completed \\
\hline
\end{tabular}


Continuation

Appendix 3. Ongoing studies characteristics

ISRCTN89785408

\begin{tabular}{|c|c|}
\hline \multirow[t]{5}{*}{ Contact information } & Name: Dr Alasdair Santini \\
\hline & Address: not reported \\
\hline & Telephone: +44 (0)151330 2071 \\
\hline & E-mail: not reported \\
\hline & Affiliation: Royal Liverpool \& Broadgreen University Hospital (UK) and Bio-Medical Research, Ltd. (Ireland) - provide Kneehab ${ }^{T M}$ (NMES) devices \\
\hline \multirow{6}{*}{$\begin{array}{l}\text { Acronyms and } \\
\text { abbreviations }\end{array}$} & BMl: body mass index \\
\hline & TKA: total knee arthroplasty \\
\hline & CVA: cerebrovascular accident \\
\hline & NMES: neuromuscular electrical stimulation \\
\hline & TUG: Timed Up and Go \\
\hline & SCT: Stair Climbing Test \\
\hline Title & Neuromuscular electrical stimulation of the quadriceps muscle: a novel alternative to total knee replacement in the young patient \\
\hline \multirow[t]{4}{*}{ Methods } & Study design: randomized controlled trial \\
\hline & Random sequence generation: not reported \\
\hline & Allocation concealment: not reported \\
\hline & Masking: single-blind \\
\hline \multirow[t]{4}{*}{ Participants } & Location: Dublin, Ireland \\
\hline & Target sample size (n): 35 to 40 patients (15 to 20 per study group) \\
\hline & $\begin{array}{l}\text { Inclusion criteria: male and female patients aged } 45 \text { to } 55 \text { years old; grade } 3 \text { to } 4 \text { arthroscopically diagnosed knee arthritis; conservatively managed; } \\
\text { ambulatory patients }\end{array}$ \\
\hline & $\begin{array}{l}\text { Exclusion criteria: morbid obesity }(\mathrm{BMI}>40) \text {; uncontrolled hypertension; anticoagulant therapy; neurological disorder; other lower limb impairment } \\
\text { affecting function including amputation; malignancy; inflammatory arthritis; implanted pacemaker or defibrillator; dermatological conditions affecting the } \\
\text { thigh; recent participation in an exercise or strength training program; inability to walk unassisted }\end{array}$ \\
\hline \multirow[t]{2}{*}{ Interventions } & $\begin{array}{l}\text { NMES group: participants will receive specific instruction from a member of the study team on application and logbook recording of the stimulator. The } \\
\text { device will be applied to the affected thigh with electrode placement depending on thigh length and girth according to the manufacturer's guidelines. } \\
\text { They will also receive written instruction on the device controls and the NMES training program schedule. All NMES sessions will be performed with the } \\
\text { subject sitting with their knee flexed to } 60 \text { degrees. Wooden strips joined with a } 60 \text { degrees bend will be provided to all subjects to assist with positioning } \\
\text { throughout the program. They will sit with their feet flat on the ground and their toes against a wall to prevent knee extension caused by the resulting } \\
\text { quadriceps contractions, and thus permit isometric training } 5 \text { sessions per week (Monday to Friday) for } 6 \text { weeks } 20 \text { minutes in duration. Participants will be } \\
\text { instructed to use the device early in the morning, between } 8 \text { and } 10 \text { a.m., to minimize muscle fatigue that may occur after normal daily activities. A student } \\
\text { from Dublin City University will attend the participants' home on day } 8 \text { to ensure the subject is using the device appropriately as well as adhering to the } \\
\text { protocol. Telephone communication will occur weekly, on Fridays, with each subject in the intervention group to ensure they are adhering to the protocol. } \\
\text { The stimulator has a built-in log that records the total number of completed sessions, total treatment time, and average intensities reached for each channel } \\
\text { for the previous } 4 \text { sessions. We will document these readings at weeks 2, 4, and } 6\end{array}$ \\
\hline & $\begin{array}{l}\text { Control Group: subjects assigned to the Control Group will receive standard care. They will attend DCU at baseline, and weeks 3, 6, and } 12 \text { for quadriceps } \\
\text { strength assessments. All functional and clinical evaluations, as well as MRI scanning, self-report questionnaires and muscle biopsies will be performed as } \\
\text { in the intervention (NMES) group. They will also receive weekly telecommunication to answer any questions they may have in relation to the study }\end{array}$ \\
\hline \multirow[t]{16}{*}{ Results } & Primary outcomes: \\
\hline & - Function: WOMAC index \\
\hline & - Quality of life: SF-36 \\
\hline & Secondary outcomes: \\
\hline & - Knee ROM \\
\hline & - Body mass \\
\hline & - Performance outcome measures: 25-m timed walk test; timed SCT; up/down seated test \\
\hline & - Radiological evaluation \\
\hline & - Quadriceps muscle strength (Torque): Biodex Multi-joint System 3 dynamometer \\
\hline & - Complications of muscle biopsy \\
\hline & - Muscle sample analysis \\
\hline & - Fiber type distribution \\
\hline & - Percent fiber type area \\
\hline & - Regulatory protein analysis \\
\hline & - RNA isolation \\
\hline & Timing of outcomes measurement: weeks 1, 3, 6, 12 \\
\hline
\end{tabular}


Continuation

Appendix 3. Ongoing studies characteristics

\begin{tabular}{|c|c|}
\hline & ISRCTN89785408 \\
\hline \multirow[t]{5}{*}{ Starting date } & Main ID: ISRCTN50117467 \\
\hline & Date applied: 17th November 2008 \\
\hline & Last edited: 8th January 2009 \\
\hline & Status: not recruiting \\
\hline & Estimated study completion date: completed \\
\hline \multirow[t]{8}{*}{ Contact information } & Name: Mr. Joshua Chong Yew Ong \\
\hline & Address: 13 Lock-keepers Walk Royal Canal Park, Dublin, Ireland \\
\hline & Telephone: not reported \\
\hline & Website: http://www.cappagh.ie/ \\
\hline & Affiliation: \\
\hline & - Cappagh National Orthopaedic Hospital (Ireland) - facilities used to perform clinical assessments, muscle biopsies and MRI scans \\
\hline & - Dublin City University (DCU) (Ireland) - laboratories used for the analysis of the muscle samples and strength testing \\
\hline & $\begin{array}{l}\text { - Bio-medical Research (Ireland) - providing neuromuscular stimulators (Kneehab }{ }^{\top \mathrm{M}} \text { ) at no cost. No financial benefit or agreement has been received or } \\
\text { made }\end{array}$ \\
\hline \multirow{8}{*}{$\begin{array}{l}\text { Acronyms and } \\
\text { abbreviations }\end{array}$} & BMl: body mass index \\
\hline & NMES: neuromuscular electrical stimulation \\
\hline & DCU: Dublin City University \\
\hline & MRI: magnetic resonance imaging \\
\hline & WOMAC: Western Ontario and McMaster Universities Osteoarthritis Index \\
\hline & SF-36: Short Form Health Survey-36 \\
\hline & ROM: range of motion \\
\hline & SCT: Stair Climb Test \\
\hline Title & Effects of Kneehab'TM 12-week Peri-operative Total Knee Arthroplasty (KneehabTKA) \\
\hline \multirow[t]{4}{*}{ Methods } & Study design: randomized controlled trial \\
\hline & Random sequence generation: not reported \\
\hline & Allocation concealment: not reported \\
\hline & Masking: single blind \\
\hline \multirow[t]{4}{*}{ Participants } & Location: Liverpool, United Kingdom \\
\hline & Target sample size (n): 200 participants \\
\hline & $\begin{array}{l}\text { Inclusion criteria: individuals who are scheduled for elective TKA surgery; individuals who are at least } 18 \text { years of age; individuals with a BMI }<40 \text {; individuals } \\
\text { who are walking independently with or without assistive devices; must be able and willing to complete all study assessments and to be followed for the full } \\
\text { course of the study; must be able to read, write and follow instructions in English; must be able and willing to provide informed consent; must be willing } \\
\text { and able to attend for preoperative assessment }\end{array}$ \\
\hline & $\begin{array}{l}\text { Exclusion criteria: individuals who have failed the preoperative assessment; individuals with a history of foot and/or ankle pathology; individuals with a } \\
\text { history of tibial or femoral fractures; individuals with a history of underlying neurological conditions; individuals with physical conditions which would make } \\
\text { them unable to perform study procedures; individuals with a total hip replacement; individuals undergoing revision TKA of the same operated leg; pregnant } \\
\text { women or inadequate precautions to prevent pregnancy; diagnosis of a medical condition that would contraindicate treatment with the product, e.g. skin } \\
\text { lesions at electrode site; individuals with an active implanted medical device (i.e. pacemaker, pump); individuals with a history of stroke; individuals with a } \\
\text { history of neurological disorder that affects lower extremity function (stroke, peripheral neuropathy, Parkinson's disease, multiple sclerosis etc.); individuals } \\
\text { with a diagnosis of inflammatory arthritis (including rheumatoid arthritis, gout or psoriatic arthritis); individuals with muscle disease (i.e. muscular dystrophy); } \\
\text { visible skin injury or disease on their legs; principal investigator for this study, or member of study staff }\end{array}$ \\
\hline \multirow[t]{2}{*}{ Interventions } & $\begin{array}{l}\text { NMES group: Kneehab }{ }^{\top M} \text { on the quadriceps of the affected leg, } 20 \text { minutes, twice per day, } 5 \text { days per week, over } 12 \text {-week intervention ( } 6 \text { weeks before } \\
\text { surgery, } 6 \text { weeks after surgery) }\end{array}$ \\
\hline & Control Group: the Control Group will complete the standard physiotherapy care pre- and post-TKA surgery without NMES \\
\hline \multirow[t]{8}{*}{ Results } & Primary outcomes: \\
\hline & - Quadriceps activation \\
\hline & - Quadriceps muscle strength (Torque): 3 dynamometer \\
\hline & - Function: TUG and SCT \\
\hline & Secondary outcomes: \\
\hline & - Quality of life: SF-12 \\
\hline & - Function and quality of life: WOMAC \\
\hline & Timing of outcomes measurement: 6 weeks preoperative and 6, 12 and 52 weeks postoperative \\
\hline \multirow[t]{5}{*}{ Starting date } & Main ID: NCT01096524 \\
\hline & First received: March 24, 2010 \\
\hline & Last updated: August 7, 2013 \\
\hline & Status: not recruiting \\
\hline & Estimated study completion date: completed \\
\hline
\end{tabular}


...Continuation

Appendix 3. Ongoing studies characteristics

\begin{tabular}{|ll}
\hline Contact information & Name: Dr. Alasdair Santini \\
& Address: not reported \\
& Telephone: not reported \\
& Contact: Bio-Medical Research, Ltd. \\
& Royal Liverpool and Broadgreen University Hospitals NHS Trust \\
& Affiliation: Bio-Medical Research, Ltd.; Royal Liverpool and Broadgreen University Hospitals NHS Trust \\
& BMl: body mass index \\
Acronyms and & TKA: total knee arthroplasty \\
abbreviations & NMES: cerebrovascular accident \\
& TUG: Time Up and Go test \\
& SCT: Stair Climbing Test \\
SF-12: Short Form Health Survey-12 \\
WOMAC: Western Ontario and McMaster Universities Osteoarthritis Index
\end{tabular}

NCT01548040

\begin{tabular}{|c|c|}
\hline Title & $\begin{array}{l}\text { Compare the Effects of a Standard Therapy Protocol With a 12-week Peri-Operative Program of Kneehab XP Neuromuscular Electrical Stimulation Compared to a } \\
\text { Control Treatment in Patients Undergoing Total Knee Replacement }\end{array}$ \\
\hline \multirow[t]{4}{*}{ Methods } & Study design: randomized controlled trial \\
\hline & Random sequence generation: not reported \\
\hline & Allocation concealment: not reported \\
\hline & Masking: double-blind \\
\hline \multirow[t]{4}{*}{ Participants } & Location: Greenville, South Carolina, United States \\
\hline & Target sample size (n): 118 participants \\
\hline & $\begin{array}{l}\text { Inclusion criteria: individuals who are scheduled for TKA surgery with the Smith and Nephew Visionaire prosthesis and anterior surgical approach; individuals } \\
\text { who are at least } 40 \text { years of age; individuals with a } \mathrm{BMl}<40 \mathrm{~kg} / \mathrm{m}^{2} \text {; individuals who are walking independently with or without assistive devices; individuals with } \\
\text { a Short Performance Battery Score }>7 \text {; must be able and willing to complete all study assessments and to be followed for the full course of the study; must } \\
\text { be able to read, write and follow instructions in English; must be able and willing to provide informed consent; must be willing and able to attend the additional } \\
\text { preoperative assessment }\end{array}$ \\
\hline & $\begin{array}{l}\text { Exclusion criteria: individuals with a history of foot and/or ankle pathology; individuals with a history of tibial or femoral fractures; individuals with a history of } \\
\text { any underlying neurological conditions; individuals with physical conditions which would make them unable to perform study procedures; individuals with a total } \\
\text { hip replacement; individuals undergoing revision TKA of the same operated leg; individuals who are pregnant; individuals diagnosed with a medical condition } \\
\text { that would contraindicate treatment with the product, e.g. skin lesions at electrode site; individuals with an active implanted medical device (i.e. pacemaker, } \\
\text { pump); individuals with a history of stroke; individuals with a history of neurological disorder that affects lower extremity function (stroke, peripheral neuropathy, } \\
\text { Parkinson's disease, multiple sclerosis etc.); individuals with a diagnosis of inflammatory arthritis (rheumatoid arthritis, gout or psoriatic arthritis); individuals with } \\
\text { muscle diseases (i.e. muscular dystrophy); individuals with visible skin injury or disease on their legs; individuals who have been committed to an institution by } \\
\text { virtue of an order issued either by the courts or by an authority }\end{array}$ \\
\hline \multirow[t]{2}{*}{ Interventions } & $\begin{array}{l}\text { Kneehab }{ }^{T M} \text { group: NMES using Kneehab XP on the affected leg, } 20 \text { minutes, twice per day, } 5 \text { days a week. Subjects will begin use of the device at } 6 \text { weeks pre- } \\
\text { operatively and continue through } 6 \text { weeks postoperatively }\end{array}$ \\
\hline & $\begin{array}{l}\text { Control Group (TENS): quadriceps TENS (at a minimal sensory input) using Kneehab XP on the affected leg, } 20 \text { minutes, twice per days, } 5 \text { days a week. Subjects } \\
\text { will begin use of the device at } 6 \text { weeks preoperatively and continue through } 6 \text { weeks postoperatively }\end{array}$ \\
\hline \multirow[t]{3}{*}{ Outcomes } & Primary outcomes: isometric strength test \\
\hline & Secondary outcomes: pain; function; knee ROM; health economics outcomes \\
\hline & Timing of outcomes measurement: 1 week pre-operatively, 3, 6, 12, 52 week post operatively \\
\hline \multirow[t]{5}{*}{ Starting date } & Main ID: NCT01548040 \\
\hline & Study first received: February 16, 2012 \\
\hline & Last updated: March 21, 2012 \\
\hline & Status: recruiting \\
\hline & Estimated study completion date: incompleted \\
\hline \multirow[t]{5}{*}{ Contact information } & Name: Brian MD Burnikel \\
\hline & Address: not reported \\
\hline & Telephone: 864-454-0904 \\
\hline & E-mail: not reported \\
\hline & Affiliation: Bio-Medical Research, Ltd. \\
\hline \multirow{6}{*}{$\begin{array}{l}\text { Acronyms and } \\
\text { abbreviations }\end{array}$} & TKA: total knee arthroplasty \\
\hline & BMI: body mass index \\
\hline & CVA: cerebrovascular accident \\
\hline & NMES: neuromuscular electrical stimulation \\
\hline & ROM: range of motion \\
\hline & TENS: transcutaneous electrical nerve stimulation \\
\hline
\end{tabular}


Continuation

Appendix 3. Ongoing studies characteristics

NCT00224913

Title

Electrical Stimulation After Total Knee Arthroplasty

Methods Study design: randomized controlled trial

Random sequence generation: not reported

Allocation concealment: not reported

Masking: single-blind

Participants

Location: Newark, Delaware, United States

Target sample size (n): 200

Inclusion criteria: TKA for unilateral tricompartmental knee OA

Exclusion criteria: insulin dependent diabetes; neurological conditions; other lower extremity orthopedic problems that affect function; BMI >40

Interventions

NMES group: patients will be asked to participate in functional and strength testing sessions, lasting about 1 hour and 30 minutes. MRI testing will last about 30 minutes per session and will be performed zero to 2 weeks before surgery, and 3 to 4 weeks, 10 to 12 weeks, and 1 year after surgery

Control Group: patients will be asked to participate in functional and strength testing sessions, lasting about 1 hour and 30 minutes. MRI testing will last about 30 minutes per session and will be performed zero to 2 weeks before surgery, and 3 to 4 weeks, 10 to 12 weeks, and 1 year after surgery

Outcomes Primary outcomes: function

Secondary outcomes: not reported

Timing of outcomes measurement: zero to 2 weeks before surgery, 3 to 4 weeks, 6 to 7 weeks, 10 to 12 weeks, 6 months, 1 year, and 2 years after surgery

Starting date

Main ID: NCT00224913

First received: September 21, 2005

Last updated: October 11, 2005

Last verified: October 2005

Status: unknown

Estimated study completion date: concluded

Contact Information Name: Lynn Snyder-Mackler

Address: not reported

Telephone: 302-831-3613

E-mail: smack@udel.edu

Affiliation: Eunice Kennedy Shriver (NICHD)

Acronyms and TKA: total knee arthroplasty

abbreviations

OA: osteoarthritis

BMl: body mass index

NMES: neuromuscular electrical stimulation

MRI: magnetic resonance imaging

NICHD: National Institute of Child Health and Human Development

NCT01844193

Title

Early Postoperative Compex Rehab NMES Use for Total Knee Arthroplasty Patients

Methods

Study design: randomized controlled trial

Random sequence generation: not reported

Allocation concealment: not reported

Masking: double-blind

Participants

Location: Indiana, United States

Target sample size $(n): 60$

Inclusion criteria: patient is a candidate for unilateral primary TKA and has a primary diagnosis of osteoarthritis; patient is a male or non-pregnant female age 18 and older at time of surgery; patient has signed an IRB-approved, study-specific informed consent form; patient is willing and able to comply with the postoperative scheduled clinical evaluations and rehabilitation

Exclusion criteria: patient has active infection within the affected knee joint; patient requires revision surgery of a previously implanted TKA; patient is morbidly obese, defined as having a BMI $\geq 36$; patient has a neuromuscular or neurosensory deficiency, which limits the ability to evaluate the safety and efficacy of the intervention; patient has been diagnosed with a systemic disease or current life threatening illness and is not able to carry on normal activities of daily life (e.g. Paget's disease, renal osteodystrophy etc.); patient has a history of cardiac issues including myocardial infarction and/or has a pacemaker; patient is immunologically suppressed or receiving chronic steroids in excess of $5 \mathrm{mg}$ per day; patient has a recent history of substance dependency that may result in deviations from the evaluation schedule; patient is a prisoner; patient has indication for contralateral total knee arthroplasty within the evaluation window 
Continuation

Appendix 3. Ongoing studies characteristics

\begin{tabular}{|c|c|}
\hline \multicolumn{2}{|r|}{ NCT01844193 } \\
\hline \multirow[t]{2}{*}{ Interventions } & $\begin{array}{l}\text { NMES group: participants in this arm will use a Compex } x^{\circledast} \text { Rehab unit for neuromuscular electrical stimulation starting with postoperative at-home day } 1 \text { and } \\
\text { continue using the unit twice a day, every day, until a 10-week follow-up is reached. The unit produces a } 380 \text {-microsecond biphasic curve and utilizes a four phase } \\
\text { process for the treatment ("Warm-up", "Work", "Relaxation", and "Recovery") for a total treatment time of } 20 \text { minutes and } 5 \text { seconds per session. All frequencies } \\
\text { are delivered at the maximum subjective tolerable intensity. Participants will control this intensity and be asked to select a level that is tolerable although mildly } \\
\text { uncomfortable; they will be instructed to increase this intensity as tolerated }\end{array}$ \\
\hline & Control Group: not reported \\
\hline \multirow[t]{3}{*}{ Outcomes } & Primary outcomes: change in quadriceps force \\
\hline & $\begin{array}{l}\text { Secondary outcomes: change in functional measurements of the quadriceps; change in pain medication requests; track narcotic/pain medication prescriptions } \\
\text { for comparison between groups }\end{array}$ \\
\hline & Timing of outcomes measurement: 2-week, 6-week, 10-week, and 1-year postoperative measurements \\
\hline \multirow[t]{6}{*}{ Starting date } & Main ID: NCT01844193 \\
\hline & First received: January 11, 2013 \\
\hline & Last updated: April 26, 2013 \\
\hline & Last verified: April 2013. \\
\hline & Status: recruiting \\
\hline & Estimated study completion date: incompleted \\
\hline \multirow[t]{5}{*}{ Contact information } & Name: Frank R Kolisek \\
\hline & Address: Greenwood, Indiana, United States, 46143 \\
\hline & Telephone: 3178845200 \\
\hline & Email: fkolisek@orthoindy.com \\
\hline & Affiliation: Orthopaedic Research Foundation and DJO Incorporated \\
\hline \multirow{4}{*}{$\begin{array}{l}\text { Acronyms and } \\
\text { abbreviations }\end{array}$} & TKA: total knee arthroplasty \\
\hline & IRB: Institutional Review Board \\
\hline & BMI: body mass index \\
\hline & NMES: neuromuscular electrical stimulation \\
\hline
\end{tabular}

